\title{
Tracheobronchial Angle Measurements in Children: An Anthropometric Retrospective Study With Multislice Computed Tomography
}

\author{
Duygu Herek ${ }^{1}$ Ozkan Herek ${ }^{2} \cdot$ Furkan Ufuk $^{1}$ \\ Departments of ${ }^{1}$ Radiology and ${ }^{2}$ Pediatric Surgery, Pamukkale University School of Medicine, Denizli, Turkey
}

Objectives. The purpose of this study is to investigate if any change exists in the values of tracheal bifurcation angles (subcarinal angle [SCA] and interbronchial angle [IBA]), right and left bronchial angles (RBA and LBA) in different pediatric age groups.

Methods. Chest computed tomography (CT) images of children aged 18 years and younger were reviewed retrospectively by two radiologists who were blinded to each other's measurements. One hundred and eighteen children were involved. RBA, LBA, SCA, and IBA were measured on coronal reformatted images. Subjects were classified into three groups according to their age. Measurement of IBA was done by measuring the angle between the lines drawn along the central axis of right and left main bronchi over their length. RBA and LBA were measured at the intersection points of the lines drawn along the inferior borders of the right and left main bronchi and the line passing through the longitudinal axis of trachea. Sums of RBA and LBA gave SCA. Interobserver agreement was also analyzed.

Results. SCA, IBA, and RBA values were statistically significant between children of ages less than 10 years and over 10 years $(P<0.01)$. Interobserver agreement was excellent with an intraclass correlation coefficient score of $0.87(95 \%$ confidence interval) for RBA, SCA, and IBA measurements.

Conclusion. We concluded that tracheal bifurcation angles are wider in children of age 10 years and younger. As age increases values of SCA, IBA, and RBA decrease.

Keywords. Trachea; Spiral Computed Tomography; Thorax; Child

\section{INTRODUCTION}

Studies about tracheal bifurcation angle (tracheobronchial angle) measurements mostly involve adults or adults and children together [1-6]. Some researchers used plain chest radiograms, some used helical computed tomography (CT) and some used cadavers for calculations. Almost all of these studies revealed significant variations and discrepancies in the measurements of tracheobronchial angle, tracheal length, right and left main

- Received January 21, 2015

Revised March 8, 2016

Accepted April 19, 2016

- Corresponding author: Duygu Herek

Department of Radiology, Pamukkale University School of Medicine,

Kinikli Kampusu, 20100 Denizli, Turkey

Tel: +90-532-658-3946, Fax: +90-258-213-4922

E-mail: dtherek@yahoo.com bronchial angles, chest diameters, tracheal and bronchial diameters [1-9]. Accurate knowledge of tracheobronchial anatomy is important in surgical approaches to thoracic cavity and for application of various airway maneuvers in anesthesiology as endotracheal intubation and in bronchoscopic removal of foreign body aspiration. It is reported that $3 \%$ of all unintentional deaths among children ( $<15$ years old) were secondary to aspiration of objects into the respiratory tract and toddlers seem to be the most vulnerable for foreign body aspiration ( $77 \%$ of deaths) $[10,11]$. Some studies about the foreign body aspiration in children under 3 years of age reported that many of the foreign bodies were located in the left main bronchus [12-14]. Some other studies established no significant difference in the location of foreign bodies being either in the left or right bronchi among small children $[7,15]$. We assumed that tracheobronchial angle differences might be an explanation to aspiration site in children

Copyright (c) 2017 by Korean Society of Otorhinolaryngology-Head and Neck Surgery.

This is an open-access article distributed under the terms of the Creative Commons Attribution Non-Commercial License (http://creativecommons.org/licenses/by-nc/4.0)

which permits unrestricted non-commercial use, distribution, and reproduction in any medium, provided the original work is properly cited. 
but literature about the measurements of tracheobronchial angle in children is limited. Kubota et al. [16] measured tracheal bifurcation angle on chest radiograms in infants and children between 0-13 years of age and found mean angle value as approximately $80^{\circ}$. They reported that in adults and children not much difference exists in angle values and right bronchial angle (RBA) was always narrower than the left bronchial angle (LBA). There is a standard teaching in the literature that right main bronchus is wider than left bronchus and has a vertical angle with the trachea. However, Adriani and Griggs [17] reported that children age 3 years and less had tracheal bifurcation angle of approximately $110^{\circ}$ and both bronchial angles were equal and $55^{\circ}$. Based on these reports we suggest that bronchial angles and tracheal bifurcation angles (interbronchial angle [IBA] and subcarinal angle [SCA]) in children might have different values compared to the adults. So, we aim to investigate whether a change exists in the values of RBA, LBA, SCA, and IBA among children of different age groups.

\section{MATERIALS AND METHODS}

\section{Study population}

This study was approved by the Institutional Ethics Committee. Database of the Radiology Department was searched between November 2015 to September 2014 by entering the search terms "patients age 18 and younger, computed tomography, thorax." Exclusion criteria from the study were any disease or lesions in the mediastinum, congenital heart diseases, anatomical abnormalities like rotoscoliosis, vascular anomalies or any previous thoracic surgery that may affect the normal measurement of tracheal bifurcation angles. Sixty-seven boys and 51 girls with total number of 118 patients were included in the study. We divided the patients into three groups according to their ages. First group (group 1) consisted of children between 0-3 years of age $(n=37)$, second group (group 2) consisted of children between the ages of 4 and 10 years $(n=35)$ and the third group (group 3 ) consisted of children between the ages of 11 and 18 years $(\mathrm{n}=46)$. Mean ages (mean $\pm S D)$ in groups 1,2 , and 3 were $1.12 \pm 1.0,6.9 \pm 1.8$, and $14.4 \pm 1.7$ years, respectively.

\section{\begin{tabular}{l|l|l|l|l|l|l|l|l|l}
\hline H & I & G & H & L & I & G & H & T & S
\end{tabular}}

- Subcarinal, interbronchial and right bronchial angles are wider under 10 years of age.

- Right bronchial angle gets narrower than left bronchial angle as age increases.

- Mean subcarinal angle values are calculated over $80^{\circ}$ in children younger than 10 years old.

- Mean interbronchial angle values are calculated over $85^{\circ}$ in children younger than 10 years old.
CT examination and tracheal bifurcation angle measurement

Chest CT examinations which were performed with a 16-slice helical CT scanner (Brilliance; Philips Medical Systems, Cleveland, OH, USA) were transferred to a workstation (MxViewexp; release 4.01, Philips Medical Systems). Parameters of CT scans were as follows: collimation, $1.25 \times 16 \mathrm{~mm}$; pitch, 0.9 ; matrix, $512 \times 512$; tube voltage, $90 \mathrm{kV}$ for patients under 11 years old and $120 \mathrm{kV}$ for patients over 10 years old, tube current changed automatically according to tube modulation system. Slice thickness was $5 \mathrm{~mm}$ for transverse images and reconstructed series were obtained. IBA, SCA, RBA, and LBA measurements were performed on reformatted coronal images by two radiologists separately: one with 14-year experience and other with 5-year experience on chest $\mathrm{CT}$, at a window setting wider than a normal mediastinal window setting (window width, 500; window level, -100).

We used a measurement model that has been described in details in the literature for IBA $[1,2,8]$. Measurement of IBA was done by drawing lines along the central axis of right and left main bronchi over their length and by measuring the angle formed between the intersection of these lines. Measurements of RBA and LBA were done according to the method described in several reports $[5,9,16]$. A line was drawn through the longitudinal axis of the trachea. The angles between the line that was drawn through the inferior border of right main bronchus and the line that was drawn through the longitudinal axis of the trachea was noted as RBA and for LBA the same was applied to the left bronchi. SCAs were described as the angles measured at the intersection points of the lines drawn along the inferior borders of the right and left main bronchi. Sums of RBA and LBA gave the SCA values.

\section{Statistical analysis}

Kruskal-Wallis test was performed to compare the differences between the three age groups. Mann-Whitney $U$-test was used for comparison of differences between groups 1 and 2, 1 and 3, 2 and 3. Statistical significance was defined as a $P$-value of less than 0.05. SPSS ver. 17.0 (SPSS Inc., Chicago, IL, USA) was used. Intraclass correlation coefficient (ICC) scores with a $95 \%$ CI were used to assess the agreement of the CT measurements between observers. Interobserver agreement was categorized as follows: $0-0.20$, poor; $0.21-0.40$, fair; $0.41-0.60$, moderate; $0.61-0.80$, substantial; and $0.81-1.00$, almost perfect [18].

\section{RESULTS}

We found no relationship between tracheal bifurcation angles (SCA, IBA) and gender $(P=0.847$ for SCA and $P=0.791$ for IBA). Mean SCA and IBA values and comparison of values between three age groups were given in Table 1 for both radiologists. There was no statistically significant difference between 
Table 1. Comparison of RBA, LBA, SCA, and IBA values between 3 age groups for each radiologist

\begin{tabular}{|c|c|c|c|c|c|c|c|c|}
\hline \multirow{2}{*}{ Group } & \multicolumn{4}{|c|}{ Radiologist 1} & \multicolumn{4}{|c|}{ Radiologist 2} \\
\hline & $\mathrm{RBA}\left({ }^{\circ}\right)$ & $\operatorname{LBA}\left({ }^{\circ}\right)$ & $\operatorname{SCA}\left({ }^{\circ}\right)$ & $\operatorname{IBA}\left({ }^{\circ}\right)$ & $\operatorname{RBA}\left({ }^{\circ}\right)$ & $\operatorname{LBA}\left({ }^{\circ}\right)$ & $\operatorname{SCA}\left({ }^{\circ}\right)$ & $\operatorname{IBA}\left({ }^{\circ}\right)$ \\
\hline $1(0-3 y r)$ & $41.3 \pm 6.6^{*}$ & $42.4 \pm 7.3$ & $83.7 \pm 13^{*}$ & $87.7 \pm 13^{*}$ & $41.7 \pm 6.9^{*}$ & $42.7 \pm 7.3^{*}$ & $84.4 \pm 12^{*}$ & $88.1 \pm 13^{*}$ \\
\hline $2(4-10 y r)$ & $40.2 \pm 6.4^{*}$ & $41.6 \pm 5.6$ & $81.8 \pm 11^{*}$ & $85.5 \pm 10^{*}$ & $40 \pm 6.5^{\star}$ & $42.2 \pm 5.9^{*}$ & $82.2 \pm 11^{*}$ & $86.7 \pm 13^{*}$ \\
\hline 3 (>10 yr) & $36.7 \pm 5.9$ & $40.4 \pm 5.3$ & $77.1 \pm 9.4$ & $80.8 \pm 9$ & $35.7 \pm 5.5$ & $41.1 \pm 5.3$ & $76.8 \pm 8.8$ & $80 \pm 10$ \\
\hline
\end{tabular}

Values are presented as mean \pm standard deviation.

RBA, right bronchial angle; LBA, left bronchial angle; SCA, subcarinal angle; IBA, interbronchial angle.

${ }^{*} P<0.01$ compared with group 3 .

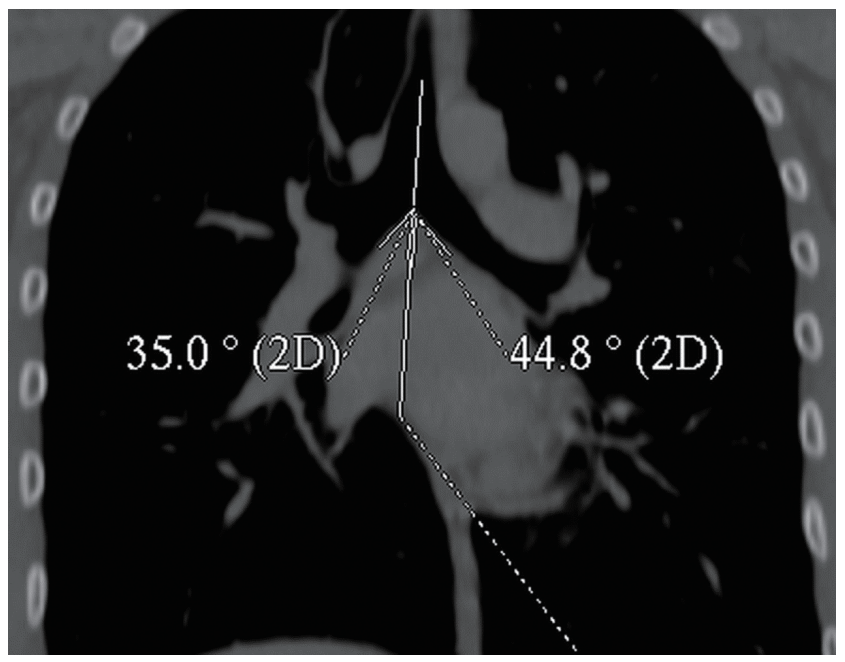

Fig. 1. Measurement of right and left bronchial angles on a coronal reformatted computed tomography image of a 16-year-old boy. Sum of these angles gives subcarinal angle. 2D, 2 dimensional.

groups 1 and 2 ( $P=0.580$ for SCA and $P=0.499$ for IBA) but there was a significant difference in the measurement of the angles between group 3 and groups 1 and $2(P<0.01)$. The measurements of SCA and IBA between radiologist 1 and 2 for each age group did not differ significantly. The tracheal bifurcation angles got wider under the age of 10 . Mean values of RBA and LBA for all age groups for both radiologists were also given in Table 1. Interobserver agreement for measurement of each angle was excellent, with an ICC score of 0.87 (95\% CI). Fig. 1 shows the measurement of RBA and LBA on a coronal reformatted CT image of a 16-year-old boy, sum of the two gave SCA. Fig. 2 shows the location of IBA and SCA on a coronal reformatted CT image of a 1-year-old boy.

\section{DISCUSSION}

In this retrospective study we measured several angles of the tracheobronchial tree in different pediatric age groups. We classified children according to their ages in which the first group counted for infants and toddlers ( $0-3$ years of age), second group counted for preschool and school age (4-10 years of age) and third group counted for pre-teens and teenagers (11-18
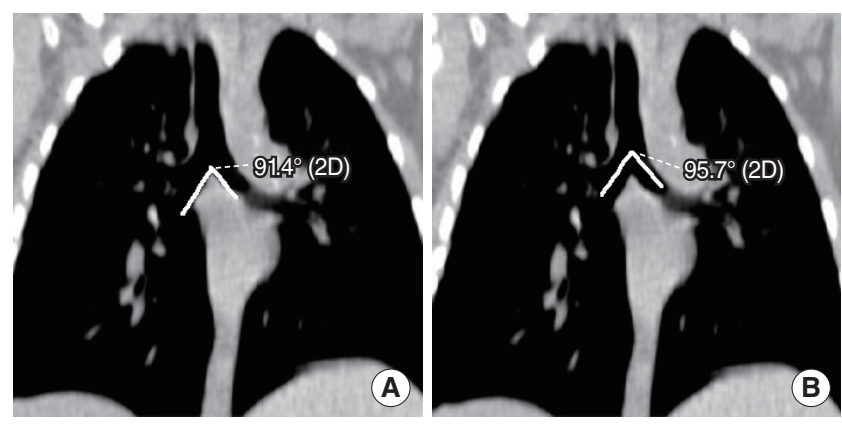

Fig. 2. Note the location of subcarinal angle (A) and interbronchial angle $(B)$ on coronal reformatted computed tomography images of a 1-year-old boy.

years of age). According to results obtained with this classification this retrospective study revealed that tracheal bifurcation angles (RBA, SCA, and IBA) in children under the age of 18 have slight differences in different age groups. But this difference is statistically significant only between children age over 10 years and under 10 years. SCAs and IBAs got wider under the age of 10 . Both radiologists measured mean SCA values over $80^{\circ}$ and IBA values over $85^{\circ}$ for children under 10 years old, but measured mean SCA as $77.1^{\circ}$ and $76.8^{\circ}$ and IBA as $80.8^{\circ}$ and $80^{\circ}$ for children of age older than 10 years. RBA measurements were also statistically significant between group 3 and the other two age groups. According to our study RBA and LBA values were smaller in children of age older than 10 years. The values of RBA got even smaller when compared to LBA and this narrowing in RBA was statistically significant between children of age greater than 10 and under 10 years of age (Table 1). Interobserver agreement was excellent and the differences in RBA, SCA and IBA values between group 3 and the other two groups were statistically significant $(P<0.01)$.

As the age increases SCA, IBA, and RBA became narrower. Our finding correlates with the study of Chunder and Guha [4] in which they reported that SCA gets narrower as the age increases. But their study included both children and adults and they did not classify children according to their age. Kubota et al. [16] measured the angles in children with different age. They included children between 0-13 years of age and they classified the children into two groups as intubation group and non-intubation group and observed a little difference in RBA and LBA 
in all ages sum of which makes tracheal bifurcation angle (SCA). Their measurements showed that SCAs ranged between $72^{\circ}$ and $87^{\circ}$ in non-intubation group and between $81^{\circ}$ and $88^{\circ}$ in intubation group almost in all ages. These results are similar to ours but they did not mention if any difference was observed between any age group. They also found that RBAs got narrower than the LBAs. In the current study we have found significant difference in the values of SCA, IBA, and RBA between the first two groups including children with 0-10 years of age and the third group with children over 10 years of age. Tahir et al. [7] found that right main bronchus was steeper in children of age 3 years and younger which was significant compared to children of age over 3 years. They calculated the mean carinal angle that corresponds to SCA in our study, as $71^{\circ}$ in children age 3 years and younger and $67^{\circ}$ in children age over 3 years which were smaller than our calculations but also showed a decrease by aging as seen in our study. Unlike Tahir et al. [7] we found that RBAs got narrower as the age increases which correlates with most findings in the literature $[5,16,17]$. According to this finding we can say that RBA gets much narrower than LBA and approaches to midline by aging. Many studies in literature refer to the fact that tracheal bifurcation angle has a wide range with many different values either in children or adults. As reported in a study done with adults, RBA which is the angle between the long axes of right main bronchus and trachea is smaller than the left main bronchial angle [5]. We also observed similar results in children over 10 years of age and we think that this finding might be the explanation to the narrowing of tracheal bifurcation angle with age. Tracheobronchial angles in adults were reported to be greater in women than in men $[2,9]$. But we found no relationship between sex and angles in children. Kamel et al. [6] reported that mean SCA values were $76^{\circ}$ in men and $81^{\circ}$ in women without any correlation with age. These results are similar to our results which we have calculated in children age over 10 years but we did not classify them according to gender. Chunder and Guha [4] also found that SCA decreases with age. Their study group also included children and the highest SCA value was $64^{\circ}$ and $61^{\circ}$ in males and females respectively. Importance of tracheal bifurcation angles is reported to be in relation with pulmonary physiology, thoracic surgery and endotracheal intubation in anesthesiology [5]. Intubation or bronchoscopic intervention of children or providing medical equipment for airway management in children is different from adults and knowledge of tracheobronchial anatomy is important for clinicians in their thoracic approaches. Kubota et al. [16] showed in their study that the RBAs were narrower than LBAs and in all their cases endotracheal tubes entered directly into the right main bronchi. Our study also proved this fact. By aging RBA gets narrower and approaches to midline making the right bronchus become almost a continuation of the trachea. In older children and adults this characteristic causes a predilection for foreign body aspiration directly into right main bronchus but in children age
10 years and younger wider SCA, IBA, RBA, and LBA values (RBA and LBA values are observed almost close to each other in this age group) might facilitate the aspiration of foreign body into left main bronchus or both main bronchi equally. Adriani and Griggs [17] also stated that RBA and LBA in little children are almost equal to each other and that LBA is more obtuse compared to RBA in adults. They reported that it would be interesting to determine the age in children at which the adult relationship of the bronchial angles begin. According to our findings bronchial angles became similar to adults after 10 years of age when RBA became significantly narrow compared to children of age under 10 years. This could be the answer to the question that Adriani and Griggs [17] wanted to explore.

This study has several limitations. One limitation is that we did not calculate any other variables as tracheal length and width, bronchial lengths, width or volume of thoracic cavity. Second, we did a retrospective study and did not know if any patient had anesthesia during CT examination. In our institute mostly anesthesia is not preferred for CT even though in children of age $<3$ years, but if it is needed oral sedative solution as chloral hydrate that is prepared by pediatricians is given to babies with feeding bottle. We don't know if sedation would affect tracheal bifurcation angle measurements. Finally, inspiration level could not be controlled by any means in little children especially under the age of 5 . But, as we classified these children mostly into one group (group 1), this limitation applies equally to all children in that group.

According to our results we concluded that tracheal bifurcation angles (SCA and IBA) are wider in children of age 10 years and younger. RBAs get narrower than LBAs as age increases. These findings about RBA and bifurcation angles may contribute to aspiration of foreign body into both bronchi equally or into left main bronchus rather than right in little children and also to the fact how endotracheal tubes accidentally enter into right main bronchus when erroneously pass the carina. However further clinical studies with children who aspirated foreign bodies and with children and adults in whom the endotracheal tubes are placed accidentally in main bronchi should be planned to prove these findings.

\section{CONFLICT OF INTEREST}

No potential conflict of interest relevant to this article was reported.

\section{REFERENCES}

1. Haskin PH, Goodman LR. Normal tracheal bifurcation angle: a reassessment.AJR Am J Roentgenol. 1982 Nov;139(5):879-82.

2. Karabulut N. CT assessment of tracheal carinal angle and its deter- 
minants. Br J Radiol. 2005 Sep;78(933):787-90.

3. Chunder R, Nandi S, Guha R, Satyanarayana N. A morphometric study of human trachea and principal bronchi in different age groups in both sexes and its clinical implications. Nepal Med Coll J. 2010 Dec;12(4):207-14.

4. Chunder R, Guha R. A morphometric study of human subcarinal angle in different age groups in both sexes and its clinical implications. Indian J Basic Appl Med Res. 2015 Mar;4(2):424-30.

5. MiW, Zhang C, Wang H, Cao J, Li C, Yang L, et al. Measurement and analysis of the tracheobronchial tree in Chinese population using computed tomography. PLoS One. 2015 Apr;10(4):e0123177.

6. Kamel KS, Lau G, Stringer MD. In vivo and in vitro morphometry of the human trachea. Clin Anat. 2009 Jul;22(5):571-9.

7. Tahir N, Ramsden WH, Stringer MD. Tracheobronchial anatomy and the distribution of inhaled foreign bodies in children. Eur J Pediatr. 2009 Mar;168(3):289-95.

8. Murray JG, Brown AL, Anagnostou EA, Senior R. Widening of the tracheal bifurcation on chest radiographs: value as a sign of left atrial enlargement.AJR Am J Roentgenol. 1995 May;164(5):1089-92.

9. Daroszewski M, Szpinda M, Flisinski P, Szpinda A, Wozniak A, Kosinski A, et al. Tracheo-bronchial angles in the human fetus: an anatomical, digital, and statistical study. Med Sci Monit Basic Res. 2013 Jul;19:194-200.

10. Fong EW. Foreign body aspiration. In: Yamamoto L, editor. Case based pediatrics for medical students and residents. 2nd ed. Hono- lulu, HI: Department of Pediatrics, University of Hawaii John A. Burns School of Medicin; 2002. p. 311-4.

11. Itasca IL. National Safety Council: injury facts, 2001 ed. Itasca, IL: National Safety Council; 2001.

12. Cohen SR, Herbert WI, Lewis GB Jr, Geller KA. Foreign bodies in the airway: five-year retrospective study with special reference to management. Ann Otol Rhinol Laryngol. 1980 Sep-Oct;89(5 Pt 1):437-42.

13. Daniilidis J, Symeonidis B, Triaridis K, Kouloulas A. Foreign body in the airways: a review of 90 cases. Arch Otolaryngol. 1977 Oct;103 (10):570-3.

14. Van Looij MA, Rood PP, Hoeve LJ, Borgstein JA. Aspirated foreign bodies in children: why are they more commonly found on the left? Clin Otolaryngol Allied Sci. 2003 Aug;28(4):364-7.

15. Baharloo F, Veyckemans F, Francis C, Biettlot MP, Rodenstein DO. Tracheobronchial foreign bodies: presentation and management in children and adults. Chest. 1999 May;115(5):1357-62.

16. Kubota Y,ToyodaY, Nagata N, Kubota H, Sawada S, Murakawa M, et al. Tracheo-bronchial angles in infants and children. Anesthesiology. 1986 Mar;64(3):374-6.

17. Adriani J, Griggs TS. An improved endotracheal tube for pediatric use. Anesthesiology. 1954 Sep;15(5):466-70.

18. Landis JR, Koch GG. The measurement of observer agreement for categorical data. Biometrics. 1977 Mar;33(1):159-74. 